\title{
UMA BREVE DISCUSSÃO TEÓRICA ACERCA DO USO DE INSTRUMENTOS MATEMÁTICOS HISTÓRICOS NO ENSINO DA MATEMÁTICA
}

\section{A BRIEF THEORICAL DISCUSSION ABOUT THE USE OF MATHEMATICAL HISTORICAL INSTRUMENTS IN TEACHING MATH}

\author{
Verusca Batista Alves ${ }^{1}$ \\ Universidade Estadual do Ceará - UECE \\ Antonia Naiara de Sousa Batista ${ }^{2}$ \\ Instituto Federal de Educação, Ciências e Tecnologia do Ceará - IFCE
}

\begin{abstract}
Resumo
No ensino da Matemática, a História da Matemática surge como uma alternativa metodológica para o estudo dos conteúdos. Por meio dela, podemos também mostrar a contextualização política, econômica e social que influenciaram e contribuíram para evolução dos mais variados conceitos. Além disso, a História da Matemática, apresenta diversos caminhos que podem ser explorados em sala de aula, os quais podemos destacar, os instrumentos matemáticos históricos, que trazem em seu próprio desenvolvimento características específicas e cálculos matemáticos referentes a cada época em que foram confeccionados e utilizados. Dentre esses instrumentos matemáticos históricos, podemos destacar aqueles que eram utilizados na agrimensura, como por exemplo, o báculo de Jacob, outros usados na astronomia, como a esfera armilar, até aqueles presentes na náutica, no caso a Balestilha, e ainda aqueles mais complexos e sofisticados, como as Réguas de Cálculo. Por meio de cada instrumento matemático, é possível trabalhar a Matemática de maneira lúdica e manipulativa, tornando as aulas mais dinâmicas e atrativas para os alunos. Proporcionando assim, uma aprendizagem mais significativa e consolidada, e ainda estimulando o senso crítico dos mesmos por meio do contexto histórico no qual os conceitos matemáticos foram originados e desenvolvidos. No intuito de contribuir com o tema proposto, apresentamos neste trabalho uma reflexão acerca das contribuições do uso desses instrumentos matemáticos históricos em sala de aula. Apontamos algumas propostas de uso desses instrumentos descrevendo-as sobre um propósito de traçar um pensamento inicial para o planejamento dos professores. Assim, por meio deste estudo buscamos contribuir para a compreensão das potencialidades que os instrumentos matemáticos históricos fornecem para a Educação Matemática.
\end{abstract}

Palavras-chave: Ensino da Matemática, História da Matemática, Instrumentos Matemáticos Históricos.
Abstract
${ }^{1}$ E-mail: verusca.alves@aluno.uece.br.
${ }^{2}$ E-mail: antonianaiarabatista@yahoo.com.br. 
In the field of Mathematics teaching, the History of Mathematics emerges as an alternative methodology for the study of the contents. Through it, we can also show the political, economic and social context that influenced and contributed to the development of various concepts. Moreover, the History of Mathematics has several paths that can be explored in the classroom, which we can highlight the mathematical historical instruments that bring, in their own development, specific characteristics and mathematical calculations referring to each time that they were made and used. Among these mathematical historical instruments, we can highlight those that were used in the surveying, for example, Jacob's staff, others used in astronomy, such as Armillary Sphere, those present in the nautical, in this case the Backstaff and, even those more complex and sophisticated, as the Slide Rules. Through each mathematical instrument, it is possible to work with the Math in a manipulative and playful way, making classes more dynamic and attractive to the students. Thus, providing a more meaningful and consolidated learning and stimulating their critical sense through the historical context in which the mathematical concepts were originated and developed. In order to contribute with the proposed theme, we present in this work a reflection about the contributions from the use of these mathematical historical instruments in the classroom. We point out some proposals using these instruments describing them on purpose to trace an initial thought for teacher planning. Then, through this study, we aim to contribute to the understanding of the potential that mathematical historical instruments provide for Mathematics Education.

Keywords: Teaching of Mathematics, History of Mathematics, Mathematical Instruments Historical.

\section{Introdução}

No ensino da Matemática é grande a busca por recursos que auxiliem os professores na ministração dos seus conteúdos, tornando assim suas aulas mais dinâmicas e atrativas. O uso da História da Matemática em sala de aula vem sendo bastante discutida nos últimos anos no campo da Educação Matemática. Segundo Mendes (2009), ultimamente foi crescente o número de pesquisas e trabalhos com o objetivo de estudar os aspectos teóricos e práticos da história direcionando assim sua utilização para explicar os mais diversos conceitos matemáticos.

A utilização da História da Matemática pode ser realizada de diversas formas, algumas delas seriam, fazer o uso de biografias de Matemáticos, ou contextualizar a situação política, cultural e econômica do conteúdo estudado naquele momento, ou empregar problemas matemáticos antigos para resolver junto aos alunos.

A História da Matemática no âmbito do ensino oportuniza o aluno a estudar o passado e compreender acontecimentos que, deram origem a determinados conteúdos matemáticos e contribuíram para a descoberta e construção de tantos cálculos, que muitas vezes hoje se apresentam de uma maneira não muito clara, deixando assim, os 
alunos com algumas dúvidas. Baroni, Teixeira e Nobre (2004) ressaltam que a História da Matemática oferece suportes para diversas necessidades educacionais e mostra a importância do estudo da Matemática, dando ênfase de que a mesma, não se resume em apenas cálculos, mas possui uma extensa rede de conhecimentos que vão muito além.

Mostrar ao aluno uma visão mais ampla da matemática é fundamental para seu desenvolvimento crítico, principalmente dando destaque ao fato de que a matemática não nasceu pronta e acabada, mas é fruto de um processo histórico juntamente com o desenvolvimento do trabalho humano, que se constituiu no decorrer de milhares de séculos. Assim, conhecer relatos históricos relacionados à matemática, que por alguma justificativa foram importantes no passado, pode estimular o aprendizado e o progresso da matemática utilizada em sala de aula hoje (D’AMBROSIO, 1996).

Muitas vezes, professores limitam-se ao conteúdo e à sala de aula. Entretanto, quando se ensina através da História da Matemática, os alunos entram em contanto com outras culturas, conhecem novos costumes, outras línguas, tipos de escritas, entre outros, mostrando assim, uma visão mais construtiva da matemática, diante de fatos que até então eram desconhecidos.

Dentro da História da Matemática encontramos diversos caminhos a serem estudados e explorados para que, posteriormente, se possa descobrir a verdadeira relação com a matemática aplicada nas aulas. Uma dessas vertentes é o ensino através dos instrumentos matemáticos históricos, dos quais podemos destacar, aqueles voltados ao uso na agrimensura, na astronomia ou nas navegações.

Bennet (apud SAITO, 2013, p. 1152) refere-se a esses instrumentos como instrumentos matemáticos, pois afirma que os mesmos, "foram concebidos para medir aquilo que Aristóteles (1952) denominava “quantidades" (distâncias e ângulos)". Logo, o surgimento de muitos desses instrumentos ocorreu devido as necessidades que foram nascendo em diferentes períodos históricos.

Segundo Saito (2015, p. 186), nos séculos XVI e XVII, em diversas regiões da Europa, começou a se propagar oficinas destinadas a confecção de vários instrumentos matemáticos, dentre eles podemos destacar desde os mais simples, como o astrolábio, o quadrante, entre outros, até os mais complexos, no caso a régua de cálculo, que tinham como objetivo atuar na solução de diferentes problemas matemáticos da época.

Nesse período a matemática começa a ser vista não apenas com o intuito de resolver problemas de caráter prático, mas também como um componente fundamental para investigar diferentes perspectivas no âmbito da natureza (SAITO e DIAS, 2011). 
Esses instrumentos eram confeccionados por artesãos, especializados nessa área, e que muitas vezes eram conhecidos como 'praticantes de matemática', sendo que em sua maioria não possuíam formação acadêmica (SAITO, 2015, p. 187). Por isso, muitos deles se reuniam em torno de corporações de ofício para se aprimorar em relação ao seu trabalho, e desse modo divulgar esses conhecimentos de geração em geração.

Assim, muitos desses instrumentos nasceram de uma necessidade que emergiu de problemas, muitas vezes, de ordem matemática, observacional ou experimental (DAUMAS, 1989; HACKMANN, 1989; TURNER, 1998 apud SAITO e DIAS, 2011). A maioria desses instrumentos matemáticos, ao longo dos anos, foram sendo aperfeiçoados e adequados às necessidades urgentes de cada período histórico, dando lugar aos instrumentos de agrimensura, que por sua vez foram cedendo espaço aos astronômicos até se chegar aos de origem náutica.

\section{Potencialidades dos instrumentos matemáticos históricos}

São muitas as competências pedagógicas que a História da Matemática proporciona ao ensino. Sua aplicação no campo da Matemática permite um estudo concomitante entre o passado e o presente, auxiliando assim, no processo de aprendizagem de diversos conceitos matemáticos.

O ensino de Matemática, baseado no seu desenvolvimento ao longo dos anos, permite que o aluno visualize essa ciência como uma criação humana, desmistificando a disciplina. Nesse sentido, a Matemática pode ser introduzida de forma lúdica e simples através da história.

Destacamos que uma das formas de tratar desse paralelo entre o passado e o presente, é por meio do ensino através dos instrumentos matemáticos históricos. Esta linha de estudo possibilita que o aluno visualize as relações da matemática e outras ciências, auxiliando no processo de aprendizagem do assunto estudado.

Para Pereira (2015), o uso de instrumentos históricos para o ensino da Matemática auxilia no processo de construção do conhecimento. $\mathrm{O}$ ensino atrelado ao uso desses instrumentos matemáticos históricos, possibilita a extração do conteúdo matemático, cultural, social e econômico da sua época de criação.

Saito (2014) afirma que a exploração dos instrumentos através de propostas que tratem além do conteúdo matemático, mas também do conteúdo histórico, social, econômico e cultural de uma determinada época, fornecem ao aluno um conjunto de relações que auxiliam na construção do conhecimento. 
Diante do exposto, entendemos que a aplicação dos instrumentos no ensino de conteúdos matemáticos incentiva o aluno a tornar-se um ser mais crítico, refletir sobre o passado e a importância do desenvolvimento histórico dos conceitos matemáticos estudados, em relação à sociedade que vive.

A respeito dos instrumentos, Saito (2014, p.28) afirma que:

\begin{abstract}
As potencialidades didáticas e/ou pedagógicas na reconstrução de instrumentos antigos podem ser exploradas por meio de uma proposta que busque revelar não só os conhecimentos matemáticos incorporados nesses instrumentos, mas também a complexa rede de conhecimentos que "esteve" e "está" presente no processo de sua construção e uso.
\end{abstract}

Dessa forma, a construção de objetos antigos, que possuem em sua essência um significado histórico e matemático, auxiliam na contextualização dos conteúdos ensinados em sala de aula. Permitindo assim, que o professor faça uma articulação entre a história e o ensino da Matemática.

No que diz respeito aos instrumentos matemáticos, apresentamos alguns deles de maneira a classificá-los de acordo com a sua função (quadro 1):

Quadro 1: Instrumentos segundo sua classificação

\begin{tabular}{|c|c|c|}
\hline \multicolumn{3}{|c|}{ Instrumentos Matemáticos } \\
\hline Agrimensura & Astronômicos & Náuticos \\
\hline Chorobate & $\begin{array}{c}\text { Quadrante } \\
\text { astronômico }\end{array}$ & Quadrante Náutico \\
\hline Galvanômetro & Astrolábio planisférico & Astrolábio Náutico \\
\hline Groma & Esfera armilar & Balestilha \\
\hline Báculo de Jacob & Nocturlábio & Kamal \\
\hline Latino rádio & Nónio & Sextante \\
\hline
\end{tabular}

Fonte: Das autoras

Ainda que os instrumentos citados sejam todos instrumentos matemáticos, há aqueles que não se encaixam em uma categoria específica, como é o caso da Régua de Cálculo, que foi criada pelo professor Inglês William Oughtred (1574 - 1660) apenas para "finalidades práticas, visto que o seu usuário não estava preocupado com os princípios matemáticos nele incorporado, mas apenas em saber o que fazer com ele" (TURNER 1973, apud SAITO, 2011, p. 3). 
Assim, investigamos o uso de instrumentos matemáticos históricos, levando em consideração seu processo de desenvolvimento e construção, dando enfoque na História para explorar os conceitos matemáticos envolvidos, pois:

Acreditamos que a construção, a utilização e a realização das atividades propostas para o uso do instrumento, possam permitir ao aluno, visualizar as relações entre conceitos da Matemática e outras ciências, levando-o à compreensão de noções matemáticas, possibilitando, assim, a aprendizagem dos conteúdos ensinados (PEREIRA, 2015, p. 14).

Logo, podemos perceber que por meio da manipulação dos instrumentos é possível proporcionar ao aluno a interdisciplinaridade entre a Matemática e outros conceitos presentes na ciência, ampliando assim, o seu conhecimento e fomentando o seu senso crítico em relação as mais distintas áreas culturais, política e econômica.

\title{
O uso de instrumentos matemáticos históricos na sala de aula
}

Diante do trabalho com instrumentos matemáticos, notamos o quanto é importante não apresentar esses objetos históricos já finalizados para o público alvo, e sim mostrar o passo a passo de sua construção. Assim, a cada ação realizada sobre o mesmo ressalta-se o conceito matemático aplicado naquele momento.

Desta maneira, o professor tem a oportunidade de participar de cada etapa do desenvolvimento do instrumento, fazendo uma reflexão sobre como se deu sua construção, mesmo que as ferramentas disponíveis naquele período de confecção do instrumento sejam diferentes das que dispomos hoje. Entretanto,

\begin{abstract}
Para que possamos examinar um instrumento matemático de forma contextualizada, é necessário que consideremos que os instrumentos e os tratados que versam sobre eles descrevem e transmitem "materialmente" informações a respeito de seu passado. Em outros termos, tais instrumentos devem ser estudados não só em relação aos que eles são (em sua materialidade), mas também em relação a como eles foram utilizados, fabricados e disseminados. (SAITO 2013, p. 1154).
\end{abstract}

Estudar os acontecimentos de uma determinada época que influenciaram o surgimento do instrumento, a sua produção e para quais fins foram direcionados, nos ajuda a compreender o instrumento de uma maneira mais abrangente, entendendo, portanto, situações sociais, políticas, econômicas que influenciaram o surgimento do mesmo, além de conhecer uma matemática mais rústica e com recursos diferentes que possibilitaram a construção desses instrumentos matemáticos.

Além disso, Dias e Saito (2011, p. 6) afirmam que, “[...] a mobilização de conceitos na atividade pode proporcionar a reflexão do conhecimento sintetizado no 
próprio instrumento, diferente de outras abordagens didáticas com instrumento que visam somente o uso". Por isso, sempre ressaltamos a manipulação e construção do instrumento, que viabiliza ao aluno perceber a Matemática por trás do mesmo, e assim, obter uma aprendizagem mais consistente e significativa.

A utilização desses instrumentos como um recurso didático requer um planejamento de uma atividade, gerado por ações sobre o mesmo, com o intuito de despertar no aluno questionamentos, acerca do porquê de certas passagens na construção do instrumento matemático se deram dessa maneira. Para Oliveira (2010, p. 3):

Por meio do manuseio de objetos antigos o professor pode mergulhar na ação de descobrir conhecimentos e prosseguir numa viagem ao passado, na tentativa de entender o contexto cultural que os geraram, constituindo uma importante fonte para criar novas formas de abordar a evolução do conhecimento matemático.

No passado cada usuário tinha restrições quanto à maneira de realizar suas medidas com esses instrumentos matemáticos, isso nos repassa conhecimentos matemáticos presentes até mesmo na maneira de manusear os instrumentos. Saito (2013) nos lembra que o processo de medição envolvia três aspectos importantes entre si, que são: a colocação do indivíduo no ambiente, a disposição do olho quanto ao instrumento e a acomodação do instrumento no espaço.

Desta forma, vimos nos instrumentos um recurso voltado não só para o crescimento do conhecimento do aluno, mas também do professor, que primeiramente tem esse contato e consequentemente busca planejar e efetuar uma aula fazendo uso desse recurso, que são os instrumentos matemáticos históricos.

Para que o professor utilize esse recurso, é requerido primeiramente questionamentos e indagações acerca de várias etapas presentes não só na construção do mesmo, mas também em partes históricas que de algum modo provocaram alguma influência no desenvolvimento do instrumento.

Por isso, resumimos em três, as formas de utilização dos instrumentos em sala de aula:

- O professor poderá previamente confeccionar um instrumento, levá-lo para sala de aula e apresentá-lo aos seus alunos;

- O professor poderá propor aos seus alunos uma atividade de confecção, pois dependendo do instrumento escolhido, os materiais são de baixo custo e fácil acesso e o professor poderá sugerir que cada aluno providencie o próprio material; 
- Dependendo do instrumento, o professor poderá previamente confeccionar vários exemplares e, na aula destinada, distribuí-los aos alunos e promover uma atividade em grupo de manuseio do objeto.

Posteriormente iremos descrever mais sobre cada categoria e mostrar as possíveis situações que o professor poderá utilizar e explorar para o uso dos instrumentos matemáticos em relação ao ensino de conceitos matemáticos.

\section{Atividades com os instrumentos}

Em se tratando de apresentar o instrumento em sala de aula e fazer sua utilização para os fins devidos, destacamos aos professores três formas de uso de um instrumento. Deixamos claro que, para a sequência de propostas é necessária uma adequação para cada instrumento trabalhado, pois alguns são de fácil confecção e manuseio, outros envolvem uma maior complexidade, assim, como são propostas, o professor deverá adequar-se para utilizá-las.

1. Levar o material já confeccionado e apresentá-lo em sala de aula.

O professor poderá confeccionar um exemplar do instrumento e levá-lo para sala de aula, para introduzir um determinado conteúdo, podendo gerar uma curiosidade em seus alunos, pelo simples fato de estar trabalhando com um objeto manipulável em sala de aula.

Apresentar o instrumento pode despertar a curiosidade daqueles que nunca tiveram contato com objetos antigos. Porém, a sugestão vai além de apenas mostrar o instrumento em sala, pois como já mencionado antes, é importante que se planeje uma ação na qual seja possível ressaltar o conteúdo matemático através da confecção do instrumento matemático envolvido.

É necessário que em um primeiro momento, o professor possa desenvolver um passo a passo relacionado à construção, fazendo assim uma primeira apresentação do objeto, pois nesse aspecto, sua utilização representa uma prática matemática do passado, podendo em seguida, propor a construção do instrumento aos alunos.

É o caso, por exemplo, da Régua de Cálculo Circular, um instrumento cujo manuseio baseia-se na utilização dos Logaritmos, em que o professor poderá utilizá-la para tratar desse conteúdo. Ao apresentar uma aplicação prática, o professor estará contribuindo para a aprendizagem dos alunos. 
2. Trabalhar a confecção do instrumento junto aos alunos.

A confecção do instrumento junto aos alunos é uma das mais produtivas possibilidades que o professor poderá adotar. Por meio da construção o professor estará trabalhando aspectos sociais, pois permitirá a interação entre os alunos.

Esse aspecto também favorece a experimentação em que, os alunos por meio de tentativas e erros obtém o produto final, o instrumento. Tais "erros de nossos alunos podem ser interpretados como verdadeiras amostragens dos diferentes modos que os alunos podem utilizar para pensar, escrever e agir." (LORENZATO, 2006, p. 50). Por isso, a experimentação é favorável à aprendizagem, por se tratar de descobertas feitas pelos próprios alunos, em que eles investigam por meio de tentativas até obter sucesso.

Para a confecção, os alunos devem ter o conhecimento matemático necessário para as medições, marcações ou graduações que o instrumento venha a ter. Isso favorece a busca por métodos que facilitem o processo de construção, bem como o tratamento de outros conteúdos matemáticos que sejam necessários para a confecção do instrumento.

3. Levar vários exemplares confeccionados para a sala de aula.

Em se tratando do manuseio, este fornece ainda mais possibilidades ao professor, pois através das tentativas de uso do instrumento, o aluno estará imerso na investigação, analisando suas próprias dificuldades e elaborando planos de ação para o estudo através de um objeto manipulável.

Assim, o manuseio de um instrumento realizado para a resolução de exercícios propostos, instigam a investigação e possibilita que os professores, juntamente aos seus alunos, realizem a experimentação e a verificação dos resultados obtidos usando meios atuais. A exemplo disso podemos citar a construção da Balestilha, que é um instrumento náutico que foi utilizado por marinheiros para se localizarem em alto mar, por meio dos astros. A este caso, Batista (2016, p. 45) diz:

Outra sugestão de atividade seria que o educador levasse os seus alunos a um observatório para então realizar a medição entre dois astros, ou seja, encontrar a distância angular que separa um astro do outro; ou a um local que ofereça uma visão nítida do céu estrelado juntamente com a linha do horizonte visível, para então, realizar a medição angular do astro em relação à linha do horizonte. 
Após uma atividade assim, o professor poderia sugerir que os alunos verificassem os resultados obtidos através do uso da matemática atual, proporcionando uma melhor fixação do conteúdo ensinado.

\section{Considerações Finais}

A História da Matemática fornece diversas possibilidades para a Educação Matemática, onde destacamos aqui o ensino através dos instrumentos matemáticos históricos, que permite ao professor, contextualizar, justificar e demonstrar os conteúdos ensinados de forma simples e lúdica, propiciando aulas mais prazerosas e de fácil compreensão.

Segundo Pereira (2015, p. 226 apud BUSSI, 2000):

A inserção de instrumentos em sala de aula pode ocorrer de duas maneiras: visitar museus físicos e virtuais (por meio de vídeos, simuladores de computador, sites, etc.) que possuam os instrumentos ou confeccionar em sala de aula instrumentos específicos para ajudar os alunos a experimentar o trabalho matemático.

Entretanto, a opção de levar os discentes para um museu ou uma sala de informática se torna inviável em alguns momentos para o professor, pois as vezes faltam recursos financeiros na escola para levar os alunos até esses espaços culturais, ou a escola não possui uma quantidade suficiente de computadores disponíveis para todos os alunos de uma turma.

Por isso, ressaltamos nesse trabalho o uso físico do instrumento em sala de aula, como uma maneira de abordar conceitos matemáticos de forma diferenciada e instigante para o aluno, e assim consequentemente, driblando alguns possíveis contratempos que surgem para dificultar a aplicação do instrumento.

Para o professor que deseja utilizar a História da Matemática, assim como, os instrumentos matemáticos históricos, deve avaliar o nível de conhecimento da turma, para que seja possível aplicar o mesmo e assim, abordar os conceitos matemáticos de forma a não prejudicar o desenvolvimento na aprendizagem do aluno, pois toda metodologia ou recurso precisa ser adequada às necessidades de um conjunto de alunos.

Assim, os instrumentos contemplam o crescimento intelectual do aluno, bem como seu desenvolvimento crítico quando se trata da análise de um objeto antigo, que foi criado com um propósito social. Também, permite que o aluno conheça de forma íntima o desenvolvimento da matemática, e os conhecimentos incorporados nesses 
objetos, proporcionando uma análise do passado e do presente, por meio do processo da construção e manuseio do instrumento matemático.

\section{Referências}

BARONI, Rosa Lúcia Sverzut; TEIXEIRA, Marcos Vieira; NOBRE, Sergio Roberto. A investigação científica em história da matemática e suas relações com o programa de pós-graduação em educação matemática. In: BICUDO, Maria Aparecida Viggiani; BORBA, Marcelo de Carvalho. Educação matemática: pesquisa em movimento. São Paulo: Cortez Editora, 2004. p. 164-185.

BATISTA, Antonia Naiara Sousa. A balestilha: um instrumento histórico de medida para explorar conceitos matemáticos na formação inicial de professores. $2016.82 \mathrm{f}$. TCC (Graduação) - Curso de Licenciatura em Matemática, Centro de Ciências e Tecnologia, Universidade Estadual do Ceará, Fortaleza, 2016.

D'AMBROSIO, Ubiratan. Educação matemática: Da teoria à prática. São Paulo: Editora Papirus, 1996. 121 p.

DIAS, Marisa da Silva; SAITO, Fumikazu. História e Ensino de Matemática: o báculo e a geometria. In: Anais do Profmat 2011 e XII SEMINÁRIO DE INVESTIGAÇÃO EM EDUCAÇÃO MATEMÁTICA, 2011, Lisboa.

LORENZATO, Sergio. Para aprender Matemática. Campinas: Autores Associados, 2006. (Coleção formação de professores).

MENDES, Iran Abreu. Investigação histórica no ensino da matemática. Rio de Janeiro: Ciência Moderna Ltda, 2009.

OLIVEIRA, Rosalba Lopes. Artefatos históricos: mediando saberes na formação docente do professor que ensina Matemática. In: ENCONTRO NACIONAL DE EDUCAÇÃO MATEMÁTICA, 10., 2010, Salvador. Anais... Salvador: Sociedade Brasileira de História da Matemática, 2010, p. 1-11.

PEREIRA, Ana Carolina Costa. Aspectos históricos da régua de cálculo para a construção de conceitos matemáticos. São Paulo: Livraria da Física, 2015. (História da Matemática para o Ensino).

PEREIRA, Ana Carolina Costa. Construindo instrumentos históricos para o estudo de conceitos matemáticos na formação inicial de professores da UECE. In: ARAÚJO, Raphael Alves de; ARAÚJO, Rute Pereira Alves de. Pesquisa em educação: olhares múltiplos. Jundiaí: Paco Editorial, 2015. Cap. 12. p. 221-239.

SAITO, Fumikazu. Instrumentos matemáticos dos séculos XVI e XVII na articulação entre história, ensino e aprendizagem de Matemática. Rematec: História de Práticas Matemáticas, Natal, v. 16, n. 9, p.25-47, maio/ago. 2014. 
SAITO, Fumikazu. Instrumentos e o 'saber-fazer' matemático no século XVI. In: SIMPÓSIO NACIONAL DE TECNOLOGIA E SOCIEDADE, 5., 2013, Curitiba. Anais... Curitiba: 2013, p. 1151-1160.

SAITO, Fumikazu; DIAS, Marisa da Silva. História da matemática para professores: Articulação de entes matemáticos na construção e utilização de instrumentos de medida do século XVI. Natal: Sociedade Brasileira de História da Matemática, 2011.

SAITO, Fumikazu. História da matemática e suas (re)construções contextuais. São Paulo: Livraria da Física, 2015. 259 p. 